\title{
On-field Electro-ejaculation of Wild Prezwalski Horses: Results and Perspectives
}

\author{
G. Rigaux ${ }^{\text {a }}$, S. Parrilla-Hernandez ${ }^{\mathrm{b}}$, S. Egyptien ${ }^{\mathrm{b}}$, C. Gatez $^{\mathrm{b}}$, C. Carrasco-Leroy $^{\mathrm{a}}$, S. Deleuze ${ }^{\mathrm{b}}$, J. Ponthier ${ }^{* \mathrm{~b}}$ \\ ${ }^{a}$ Domaine des Grottes de Han, Han/Lesse, Belgium \\ ${ }^{\mathrm{b}}$ Equine Reproduction, University of Liège, Belgium
}

Wild horses, donkeys and zebra reproduction management is limited to natural mating by semen availability and poor knowledge of females' cyclicity. Improving electro-ejaculation could enhance semen exchange between conservation centres. However, on-field anaesthesia risks and poor quality of semen collected are limiting factors. This report aimed to describe an effective and safe on-field electro-ejaculation method and parameters of semen obtained with this method. Fertility of 3 Prezwalski horses was evaluated in a safari park. The animals were darted with acepromazine, detomidine, butorphanol and etorphine, then a balanced anaesthesia (Guaiacolate glycerol, ketamine and xylazine) was administered through a jugular catheter and oxygen was supplied by endotracheal tube. The bladder was catheterized and emptied. After 2 minutes of prostatic massage, the bovine probe of the electro-ejaculator (Minitube, Germany) was inserted in the rectum and the sequence of stimulation was performed as follows: Cycle 1: 10 stimulations $0.5 \mathrm{~V}-10$ stimulations $1.5 \mathrm{~V}-15$ stimulations $2.5 \mathrm{~V}$ - 3 minutes of rest; Cycle 2: 10 stimulations $0.5 \mathrm{~V}-10$ stimulations $1.5 \mathrm{~V}$ - 15 stimulations $3.5 \mathrm{~V}-3$ minutes of rest; Cycle 3: 10 stimulations $1.5 \mathrm{~V}$ - 10 stimulations $2.5 \mathrm{~V}$ - 10 stimulations $3.5 \mathrm{~V}$ 15 stimulations $4.5 \mathrm{~V}$ - 3 minutes of rest; Cycle $4: 10$ stimulations $0.5 \mathrm{~V}-10$ stimulations $2.5 \mathrm{~V}-10$ stimulations $3.5 \mathrm{~V}-15$ stimulations $4.5 \mathrm{~V}$. Samples were collected and analyzed separately at the end of each cycle. After the last stimulation, bladder was catheterised and presence of spermatozoa was microscopically assessed. Raw semen motility was immediately evaluated on field with a microscope on a slide maintained at $37^{\circ} \mathrm{C}$. Then samples were largely diluted with INRA96 ${ }^{\mathrm{TM}}(1 \mathrm{v} / 5 \mathrm{v})$ and sent to the lab whithin 4 hours for semen evaluation (Thoma Cell and computer assisted semen analysis (CASA)). Training of the team limited the procedure to 1 hour. No complications were observed with our anaesthetic protocol using an endotracheal tube. Higher collected numbers of spermatozoa were obtained after cycles 2 and 3 and the number of collected spermatozoa always decreased after the $3^{\text {rd }}$ cycle, low spermatozoa number was observed in urine. Onfield motilities estimated by the same experimented investigators were always lower than those of the extended semen analysed by CASA. A total of 4291,1517 and $450 \times 10^{6} \mathrm{spz}$ with respectively 56, 30 and $25 \%$ of progressive motility were obtained from the three collections. Our procedure using tele-anaesthesia, intra-venous maintenance and an endotracheal tube, which reduced the risk of aspiration pneumonia, was totally safe and effective for semen collection by electro-ejaculation in wild equids. Sufficient spermatozoa numbers were collected after three cycles of stimulations. Raw semen quality may seem low, maybe due to urinary contamination, but is improved after large dilution $(1 \mathrm{v} / 5 \mathrm{v})$ in commercial extender. Thus, semen collected from wild horses by electro-ejaculation performed under anaesthesia could be shipped after large dilution to the lab and eventually used for AI programs, if CASA results show sufficient motility. 